\title{
Reverse shoulder arthroplasty for corticosteroid-induced deltoid myopathy in a patient with systemic lupus erythematosus: a case report
}

\author{
Serkan Bayram, Ali Erşen \\ Department of Orthopedics and Traumatology, Istanbul University Faculty of Medicine, Istanbul, Turkey
}

\begin{abstract}
A 50-year-old woman who had been previously diagnosed with systemic lupus erythematosus consulted our clinic for pain and weakness in her right shoulder. On examination, she had an atrophied deltoid muscle, a painful right shoulder on movement, and a tender mass in the deltoid area. The patient was diagnosed with corticosteroid-induced deltoid myopathy, shoulder pain, and loss of range of motion that did not resolve with conservative treatment. We decided to perform reverse shoulder arthroplasty. No complications were observed at the last follow-up visit at 3 years postoperative. Unlike deltoid insufficiency that results from axillary nerve injury, deltoid myopathy due to corticosteroid use contains intact fibers. Therefore, we increased the effectivity of the remaining deltoid fibers by extending the moment arm of the anterior fibers using reverse shoulder arthroplasty and achieved reliable improvements in clinical symptoms and function without increasing the risk of dislocation.
\end{abstract}

Keywords: Reverse shoulder arthroplasty; Myopathy; Systemic lupus erythematosus; Deltoid insufficiency

Myopathy is loss of contractile function in muscles that results in weakness. Myopathies primarily cause defects within the muscle tissue and are distinct from neuromuscular junction diseases and neuropathic causes of muscle weakness [1]. The etiology of myopathy varies widely and includes inflammatory, congenital, infectious, and metabolic diseases; endocrine disorders; nutritional deficiencies; and drug-induced or toxic causes [2].

The most common type of drug-induced myopathy is corticosteroid-induced myopathy [3]. Patients with corticosteroid-induced myopathy typically present with a gradual onset of proxi- mal muscle weakness, followed by atrophy of proximal muscle groups. We report a case of corticosteroid-induced deltoid myopathy in a patient previously diagnosed with systemic lupus erythematosus (SLE) who complained of shoulder pain and immobility; she was treated with reverse shoulder arthroplasty (RSA).

\section{CASE REPORT}

This study was approved by the Academic Board of Istanbul University Istanbul Faculty of Medicine, Department of Orthopae-

Received: April 11, $2021 \quad$ Revised: June 9, $2021 \quad$ Accepted: June 14, 2021

Correspondence to: Serkan Bayram

Department of Orthopedics and Traumatology, Istanbul University Faculty of Medicine, Çapa Fatih, Istanbul 34050, Turkey

Tel: +90-212-414-2000, Fax: +90-212-410-1500, E-mail: dr.serkanbayram89@gmail.com, ORCID: https://orcid.org/0000-0001-7651-1200

Financial support: None.

Conflict of interest: None. 
dics and Traumatology (IRB No. 06.04.2020-4/2020). Informed consent was acquired from the patient prior to publieIcation of this case report.

A 50-year-old woman was admitted to the rheumatology clinic with a malar rash, multiple joint pain, and muscle weakness that began in 2015. She had no other chronic or acute comorbidities. Laboratory tests revealed a C-reactive protein level of $18 \mathrm{mg} / \mathrm{L}$ (reference range, $0-5 \mathrm{mg} / \mathrm{L}$ ), an erythrocyte sedimentation rate of $24 \mathrm{~mm} / \mathrm{hr}$ (reference range, 0-20), and a white blood cell count of $6,460 / \mu \mathrm{L}$ (normal range, $4,100-11,200 / \mu \mathrm{L}$ ). The patient was diagnosed with SLE and treated with $25 \mathrm{mg}$ of Deltacortril (prednisolone) once a day and Plaquenil (hydroxychloroquine) once a day.

During the follow-up period in the rheumatology department in 2016, the patient reported pain and weakness in her right shoulder and consulted our department. Notably, she had not undergone any previous shoulder surgery. She was examined by a senior shoulder-and-elbow surgeon. Upon examination, she had an atrophied deltoid muscle, a painful right shoulder on movement, and a tender mass in the deltoid area (Fig. 1). A shoulder range of motion examination revealed active forward flexion of $70^{\circ}$, abduction of $50^{\circ}$, internal rotation to the sacrum, and external rotation of $10^{\circ}$. The American Shoulder and Elbow Surgeons (ASES) score was 21.6 of 100, and the Constant score was 25 of 100 at preoperative evaluation. Neurological examination showed normal findings, except for slightly weak abduction and flexion in the right shoulder. The blood test results were as follows: erythrocyte sedimentation rate, $35 \mathrm{~mm} / \mathrm{hr}$; C-reactive protein, $17 \mathrm{mg} / \mathrm{L}$; and white blood cell count, 12,100/ $\mu \mathrm{L}$. For a thorough evaluation, radiography and magnetic resonance imaging (MRI) were performed. The MRI revealed substantial atro-

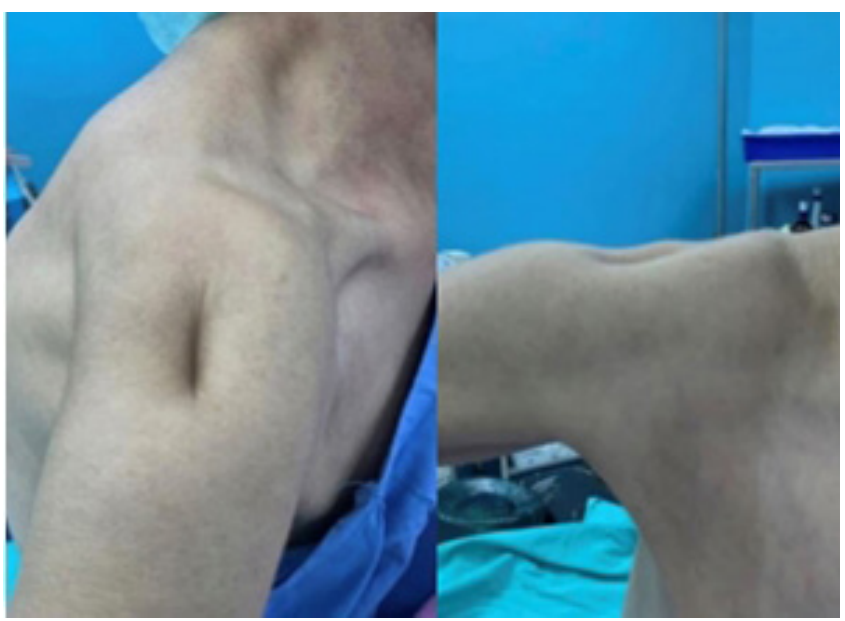

Fig. 1. The atrophied deltoid muscle was seen upon visual shoulder inspection. phy at all of the middle deltoid and most of the posterior deltoid of the right shoulder (Figs. 2 and 3). The anterior fibers of the deltoid were intact. The subscapularis and supraspinatus tendons were retracted to the medial area of the glenoid. Stage 3-4 fatty infiltration was detected in the subscapularis and supraspinatus according to the Goutallier classification [4]. Grade 2 cuff tear arthropathy was detected upon radiographic evaluation according to the Hamada classification [5].

The patient was referred to an electrodiagnostic laboratory to evaluate right shoulder weakness and atrophy; the findings were normal. However, concentric needle electromyography revealed short-term and polyphasic motor unit potentials in the musculus deltoideus upon examination with a concentric needle. Consequently, the patient was diagnosed with corticosteroid-induced deltoid myopathy, and a conservative treatment approach
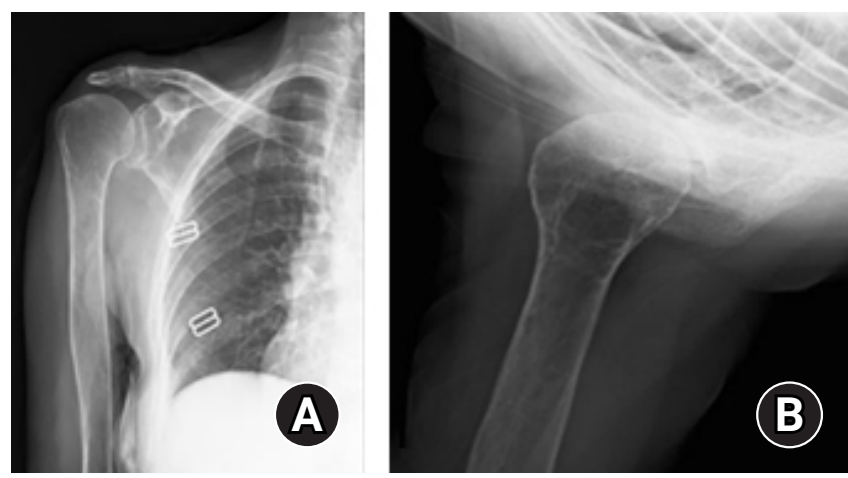

Fig. 2. (A) Preoperative anteroposterior radiograph of the patient. (B) Preoperative axillary radiographs of the patient.

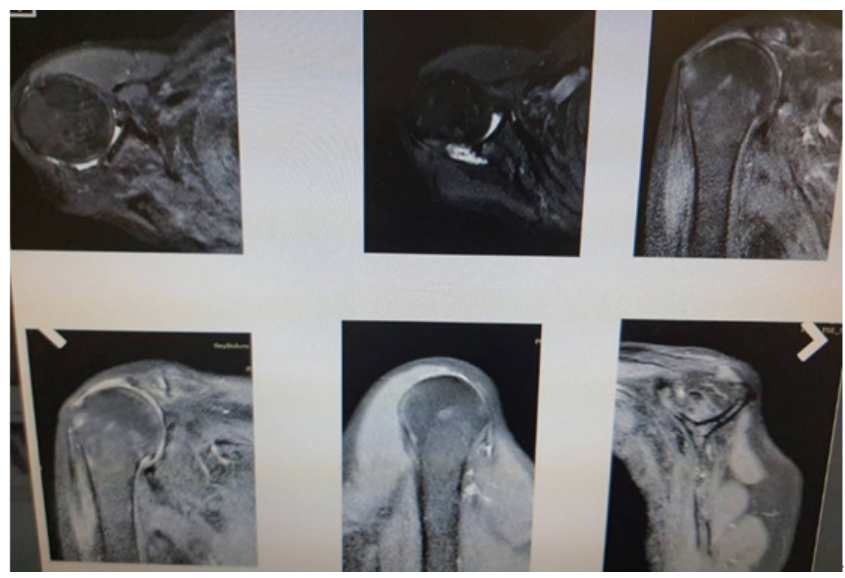

Fig. 3. Magnetic resonance imaging revealed substantial atrophy at all of the middle deltoid and most of the posterior deltoid region of the right shoulder. The anterior fibers of the deltoid were found to be intact. The subscapularis and supraspinatus tendons were retracted up to the medial area of the glenoid. Stage 3-4 fatty infiltration was detected in the subscapularis and supraspinatus according to the Goutallier classification. 
was chosen. She was followed for 6 months with symptomatic and physical therapy. However, the complaint was not resolved, and RSA was planned. Deltoid myopathy due to corticosteroid use exhibits intact fibers, unlike deltoid insufficiency due to axillary nerve injury. Therefore, RSA increased the effectivity of the deltoid fibers by extending the moment arm of the anterior fibers.

\section{Surgical Technique}

A standard deltopectoral approach was used. The long head of the biceps was identified beneath the pectoralis major tendon and incised close to its proximal insertion after rotator interval opening. Similar to MRI findings, we observed atrophic middle and posterior deltoid fibers, and the anterior fibers were intact. In addition, the supraspinatus and subscapularis muscles were retracted. The medullary cavity was located and reamed, and the head was resected (Fig. 4). Thereafter, the glenoid articular surface was exposed, and the labrum was removed. Lateralized RSA (Biomet Comprehensive Shoulder System; Zimmer, Warsaw, IN, USA) was performed. After reaming, a baseplate was implanted with a $15^{\circ}$ inferior tilt and fixed with one central screw, one inferior screw, and one superior screw, followed by implantation of a 36-mm glenosphere. Next, a size-11 uncemented humeral stem

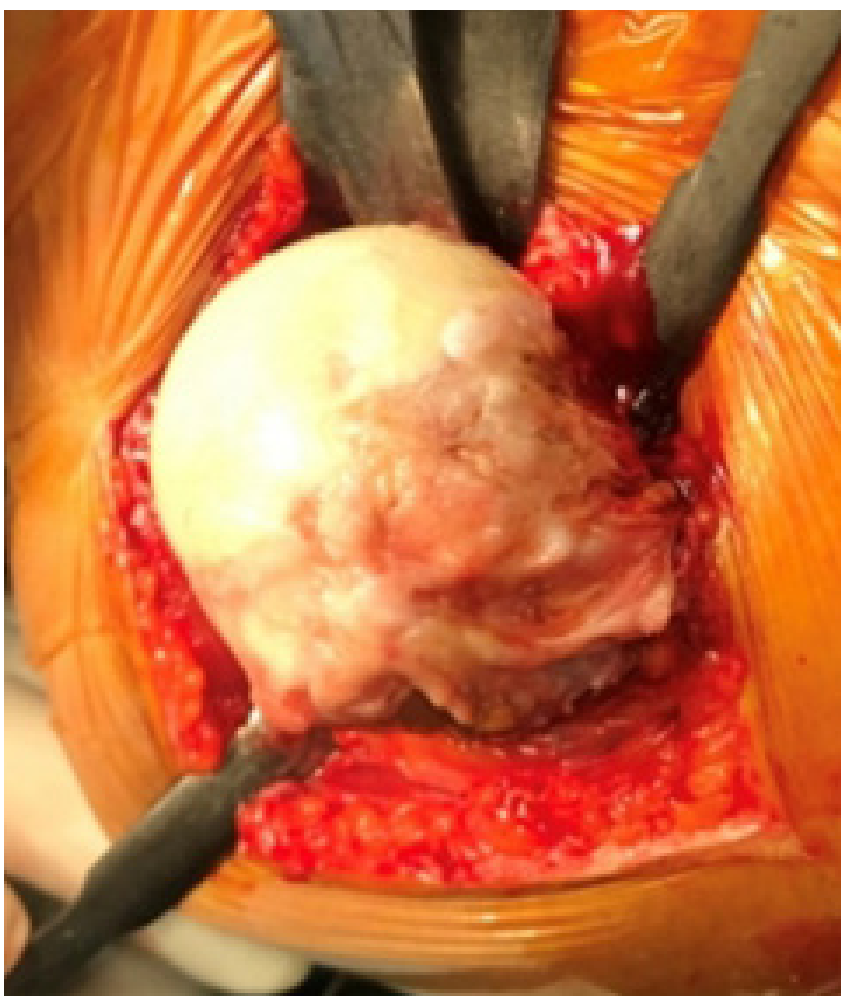

Fig. 4. Although the humeral head was healthy, the rotator cuff and deltoid muscles were torn and demonstrated insufficiency. was implanted with $20^{\circ}$ retroversion after reaming of the shaft. We then checked the range of motion and stability, and the subscapularis tendon was reattached. Soft-tissue tenodesis was performed using the biceps tendon, which was previously incised. Finally, a sling with $15^{\circ}$ abduction and neutral rotation was applied, and we performed a postoperative X-ray series (Fig. 5).

\section{Postoperative Rehabilitation}

The sling was maintained for the first 6 weeks postoperatively; however, both active and passive range of motion were permitted from the third postoperative day as long as pain allowed. At 12 weeks following surgery, strengthening exercises were introduced.

\section{Follow-up}

The patient showed no evidence of infection, fracture, or dislocation at any of the postoperative visits. No complications were observed at the last follow-up visit at three years postoperative. Moreover, the patient's active forward flexion was $160^{\circ}$, abduction was $150^{\circ}$, internal rotation was toward the upper lumbar spine, and external rotation was $30^{\circ}$. The shoulder range of motion increased significantly compared to the preoperative condition. The anterior deltoid fibers were intact at the final visit. The

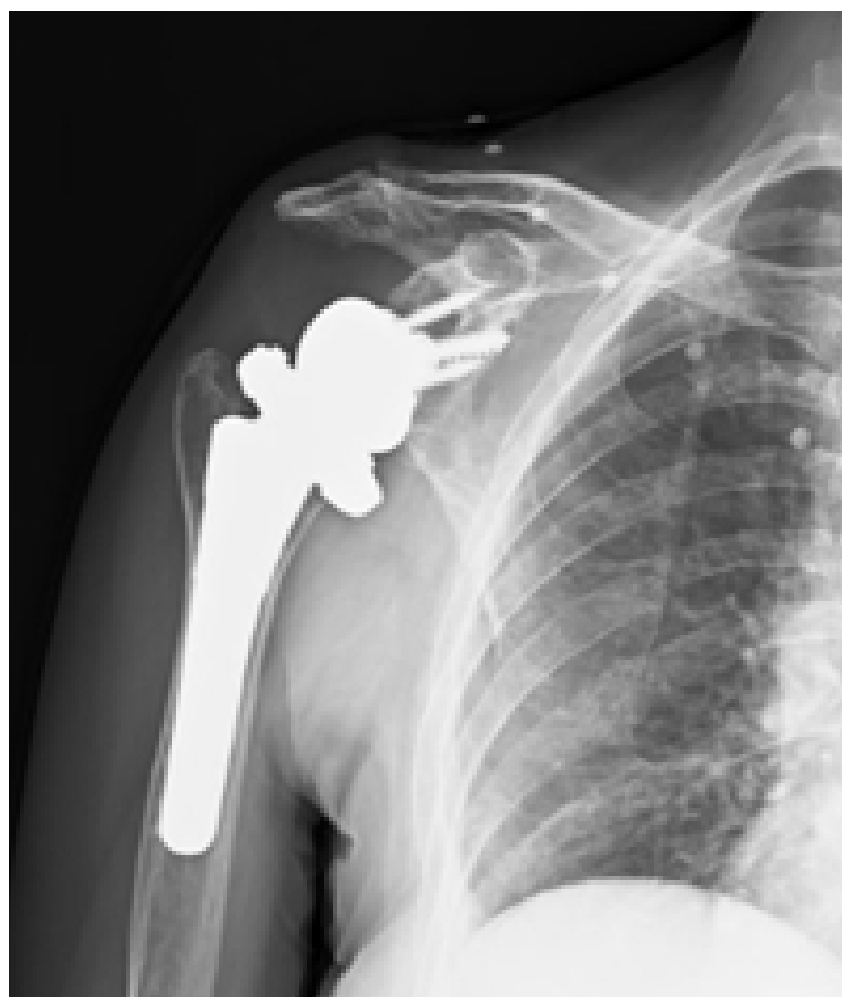

Fig. 5. Postoperative anteroposterior shoulder radiographs of the patient. 
patient was satisfied, and her ASES score was 88.3 of 100, while the Constant score was 83 of 100 .

\section{DISCUSSION}

This case study provides a unique scenario wherein RSA was used to treat successfully shoulder pain in a patient with long-standing complete deltoid insufficiency due to corticosteroid intake. We propose that deltoid insufficiency is related to as opposed to an absolute contraindication to RSA in a select patient population. In previous reports, patients with partial deltoid function had demonstrated favorable RSA outcomes [6-10]. To our knowledge, this case report is the first to present the ability of RSA to treat practically complete deltoid insufficiency (especially in the middle deltoid and posterior deltoid fibers) due to corticosteroid usage in a patient with SLE.

For patients with deltoid insufficiency, two operative procedures (either with or without muscle flap reconstruction) are available when performing RSA [4-7]. Lädermann et al. [6] reported 49 patients with deltoid impairment who were treated with RSA and followed for a mean of 38 months. They did not perform muscle flap reconstruction and observed nine postoperative complications (18\%), including two dislocations. They reported Constant score improvement from 24 to 58, Single Assessment Numeric Evaluation score of 71, and patient satisfaction rate of $98 \%$ during the final follow-up. Meanwhile, Schneeberger et al. [7] reported 19 patients with RSA who experienced unsatisfactory results following deltoid flap reconstruction because of deltoid muscle defects. In that study, 15 of 19 patients were not satisfied with the revision outcome, and 4 patients were satisfied. The mean overall subjective shoulder value was 73\% (30\%-95\%) of the normal value.

An alternative procedure uses a latissimus dorsi flap, which is recommended for patients with deltoid deficiency who are treated with RSA [8-10]. Dosari et al. [8] reported a 51-year-old male patient with a history of gunshot injury to the left shoulder that led to loss of shoulder bony and muscular structures. In that case, the patient was treated with a latissimus dorsi muscle flap because of the deltoid muscle deficiency, followed by RSA. Essilfie et al. [10] and Goel et al. [9] each reported a case where a latissimus dorsi muscle flap was used to treat deltoid muscle deficiency [8]. An RSA procedure after latissimus dorsi flap in patients with deltoid deficiency can be a successful and reproducible approach to treat such conditions.

In our patient, the shoulder center of rotation was mediatized, and the deltoid remained tensioned for RSA stability and motion. However, cases of RSA used for treating deltoid insufficiency are limited. Our case report suggests that RSA can be used to treat deltoid myopathy and can produce reliable improvements in clinical manifestations and functions without an increased risk of dislocation. The difference between this case report and other deltoid insufficiency patients treated with RSA for extending the moment arm of the anterior deltoid fibers was that our patient's deltoid insufficiency was not secondary to surgery but resulted from corticosteroid use, and the patient expected to continue using these medications postoperatively.

\section{ORCID}

Serkan Bayram

https://orcid.org/0000-0001-7651-1200

Ali Erşen

https://orcid.org/0000-0001-6241-2586

\section{REFERENCES}

1. Furst DE, Amato AA, Iorga ŞR, Gajria K, Fernandes AW. Epidemiology of adult idiopathic inflammatory myopathies in a U.S. managed care plan. Muscle Nerve 2012;45:676-83.

2. Barnes PR, Dalakas MC, Jones DH, Palace JA, Rose MR. Myopathies in clinical practice. Oxford: Isis Medical Media; 2003. p. 59-83.

3. Jakati S, Rajasekhar L, Uppin M, Challa S. SLE myopathy: a clinicopathological study. Int J Rheum Dis 2015;18:886-91.

4. Goutallier D, Postel JM, Bernageau J, Lavau L, Voisin MC. Fatty muscle degeneration in cuff ruptures: pre- and postoperative evaluation by CT scan. Clin Orthop Relat Res 1994;(304):78-83.

5. Hamada K, Fukuda H, Mikasa M, Kobayashi Y. Roentgenographic findings in massive rotator cuff tears: a long-term observation. Clin Orthop Relat Res 1990;(254):92-6.

6. Lädermann A, Walch G, Denard PJ, Collin P, Sirveaux F, Favard $\mathrm{L}$, et al. Reverse shoulder arthroplasty in patients with pre-operative impairment of the deltoid muscle. Bone Joint J 2013; 95:1106-13.

7. Schneeberger AG, Müller TM, Steens W, Thür C. Reverse total shoulder arthroplasty after failed deltoid flap reconstruction. Arch Orthop Trauma Surg 2014;134:317-23.

8. Dosari MA, Hameed S, Mukhtar K, Elmhiregh A. Reverse shoulder arthroplasty for deltoid-deficient shoulder following latissimus dorsi flap transfer: case report. Int J Surg Case Rep 2017;39:256-9.

9. Goel DP, Ross DC, Drosdowech DS. Rotator cuff tear arthropathy and deltoid avulsion treated with reverse total shoulder arthroplasty and latissimus dorsi transfer: case report and review of the literature. J Shoulder Elbow Surg 2012;21:e1-7.

10. Essilfie A, McKnight B, Heckmann N, Rick Hatch GF 3rd, 
Serkan Bayram and Ali Erşen. Reverse shoulder arthroplasty in deltoid deficit

Omid R. Revision reverse total shoulder arthroplasty in a patient with preoperative deltoid insufficiency: a case report. J Shoulder Elbow Surg 2017;26:e232-5. 